\title{
On the Blowup for the 3D Axisymmetric Incompressible Chemotaxis-Euler Equations
}

\author{
Shangyong $\mathrm{An}^{1}$ and Qian Zhang $\mathbb{D}^{2}$ \\ ${ }^{1}$ School of Languages and Culture, Hebei GEO University, Shijiazhuang 050031, China \\ ${ }^{2}$ Hebei Key Laboratory of Machine Learning and Computational Intelligence, School of Mathematics and Information Science, \\ Hebei University, Baoding 071002, China
}

Correspondence should be addressed to Qian Zhang; zhangqian@hbu.edu.cn

Received 12 January 2021; Revised 23 March 2021; Accepted 25 March 2021; Published 7 April 2021

Academic Editor: Alberto Fiorenza

Copyright (c) 2021 Shangyong An and Qian Zhang. This is an open access article distributed under the Creative Commons Attribution License, which permits unrestricted use, distribution, and reproduction in any medium, provided the original work is properly cited.

In this paper, we investigate the 3D incompressible chemotaxis-Euler equations. Taking advantage of the structure of axisymmetric fluids, we establish the blowup criterion of the system using the Fourier localization method.

\section{Introduction}

The effect of oxygen attraction on the emergence of bioconvective patterns is studied in $[1,2]$. Some experiments, such as a colony of Bacillus subtilis suspending in a drop of water, are carried out to identify this phenomenon. From paper [36], we can also find the important role of chemotaxis between sperm and eggs. The following model, in [1], is introduced to analyze the above phenomenon:

$n_{t}+u \cdot \nabla n=\Delta n+\chi \nabla \cdot\left(n \nabla(\Delta)^{-1} c\right)+\kappa n-\mu n^{2}, \quad n(x, 0), x \in d$.

Here, $n$ and $u$ represent the concentration of bacteria and the velocity field of the transported water, respectively. Besides, the vector field $u$ is divergence free and independent of $n$. The equation describes the evolution of the bacteria transported by the velocity field of the fluid. Moreover, these cells are attracted by the oxygen concentration generated by chemotaxis. For the term $\chi \nabla \cdot(n \nabla$ $\left.(\Delta)^{-1} c\right), \chi>0$ is a parameter controlling the influence of the chemotactic effect. In addition, $\kappa$ is the strength growth rate of the population and $\mu$ is a parameter regulating death by overcrowding.
Apart from Equation (1), there are a lot of other models illustrating the procedure of oxygen attraction in biology. An increasing number of mathematicians studied the process in the past years, see [7-15]. Our aim in this paper is to further explore model (1), combined with an oxygen equation and a Navier-Stokes equation, see [16]. Then, we obtain the following model in $d, d=2,3$,

$$
\left\{\begin{array}{l}
n_{t}+u \cdot \nabla n=\Delta n-\chi \nabla \cdot(n \nabla c)+\kappa n-\mu n^{2}, \\
c_{t}+u \cdot \nabla c=\Delta c-n c, \\
u_{t}+(u \cdot \nabla) u-\nabla P=\eta \Delta u-n \nabla \Phi, \\
\nabla \cdot u=0, \\
n(0, x)=n_{0}(x), c(0, x)=c_{0}(x), u(0, x)=u_{0}(x) .
\end{array}\right.
$$

The unknowns are $n, c, u$, and $P$, standing for the bacteria, the oxygen, the velocity field, and the pressure of the fluid separately. The third equation of the above system contained an extra force, buoyancy, which is produced by the density and a given gravitational potential $\Phi . \eta$ is the dissipation coefficient. If $\kappa=\mu=0$ and $\eta>0$, the global existence of weak solutions in 2 was shown in $[8,12]$. 
In this paper, we choose $\chi=\kappa=\mu=1$ and $\eta=0$, then (2) can be changed into the following one:

$$
\left\{\begin{array}{l}
n_{t}+u \cdot \nabla n=\Delta n-\nabla \cdot(n \nabla c)+n-n^{2}, \\
c_{t}+u \cdot \nabla c=\Delta c-n c, \\
u_{t}+(u \cdot \nabla) u-\nabla P=-n \nabla \Phi, \\
\nabla \cdot u=0, \\
n(0, x)=n_{0}(x), c(0, x)=c_{0}(x), u(0, x)=u_{0}(x) .
\end{array}\right.
$$

The Euler equation is shown as the following form:

$$
\left\{\begin{array}{l}
u_{t}+u \cdot \nabla u+\nabla P=0 \\
\nabla \cdot u=0 \\
u(0, x)=u_{0}(x)
\end{array}\right.
$$
form

In three dimensional space, the vorticity equation has the

$$
\omega_{t}+u \cdot \nabla \omega=\omega \cdot \nabla u
$$

But the chief difficulty is we are lacking information on the vortex-stretching term $\omega \cdot \nabla u$. Although the global existence of classical solutions for the $3 \mathrm{D}$ Euler equation is an open problem, some known results are obtained under the circumstances of axisymmetric flows without swirl. That a vector field $u$ is axisymmetric without swirl is defined as follows:

$u(t, x)=u^{r}(t, r, z) e_{r}+u^{z}(t, r, z) e_{z}, \quad x=\left(x_{1}, x_{2}, z\right), r=\left(x_{1}^{2}+x_{2}^{2}\right)^{1 / 2}$,

where $\left(e_{r}, e_{\theta}, e_{z}\right)$ is the cylindrical basis of 3 and the components $u^{r}$ and $u^{z}$ do not depend on the angular variable. With this structure, vorticity takes the form

$$
\omega=\left(\partial_{z} u^{r}-\partial_{r} u^{z}\right) e_{\theta}:=\omega_{\theta} e_{\theta}
$$

and satisfies

$$
\partial_{t} \omega+u \cdot \nabla \omega=\frac{u^{r}}{r} \omega
$$

Hence, the quantity $\Gamma:=\omega_{\theta} / r$ obeys to the equation

$$
\partial_{t} \Gamma+u \cdot \nabla \Gamma=0
$$

The goal of this paper is to build the blowup criterion of smooth solutions for (3) by the Fourier localization technique. Here, we follow ideas introduced in [17-21]. Our result reads as the following:

Theorem 1. For $s>3$, suppose the triple $\left(n_{0}, c_{0}, u_{0}\right) \in H^{s} \times$ $H^{s+1} \times H^{s+1}$ and $\Phi \in H^{s+2}$. Let $u_{0}$ be an axisymmetric divergence-free vector field and its vorticity satisfies $\omega_{0} / r \in$ $L^{3,1}$. Assume that

$$
\begin{gathered}
n \in C\left([0, T] ; H^{s}\right) \cap L^{2}\left([0, T] ; H^{s+1}\right), \\
c \in C\left([0, T] ; H^{s+1}\right) \cap L^{2}\left([0, T] ; H^{s+2}\right), \\
u \in C\left([0, T] ; H^{s+1}\right)
\end{gathered}
$$

are the smooth solutions to (3). If the condition

$$
\int_{0}^{T}\left(\|n(\tau)\|_{B_{\infty, 1}^{0}}^{2}\right) d \tau<\infty
$$

holds true, then the solutions $(n, c, u)$ can be extended beyond $T>0$.

Remark 2. In paper [20], a regularity criterion in terms of two items is established. But in Theorem 1, we give a different criterion using the only bacteria concentration in 3 . The bacteria concentration plays a more important role in this model, and the nonlinear term $-\nabla \cdot(n \nabla c)$ is difficult to estimate. Hence, using bacteria concentration to show the regularity is natural and physical.

Notation. Throughout the paper, $C$ means a harmless constant and may vary from line to line; $C_{T}$ denotes a constant $C$ relating to $T ;\|\cdot\|_{p}$ stands for the norm of the Lebesgue space $L^{p}$.

\section{Preliminaries}

In this section, we give the definition of some function spaces and recall some useful lemmas.

Firstly, we use the dynamic partition of the unity to give the definition of Besov spaces. One may check [22] for exact details. Let $\varphi \in C_{0}^{\infty}(d)$ be set in $C=\{\xi \in d, 3 / 4 \leq|\xi| \leq 8 / 3\}$ satisfying

$$
\sum_{q \in Z} \varphi\left(2^{-q} \xi\right)=1, \quad \text { for } \xi \neq 0
$$

Let $\chi(\xi)=1-\sum_{q \in N} \varphi\left(2^{-q} \xi\right)$. For $f \in S^{\prime}$, Littlewood-Paley operators are defined as follows:

$\Delta_{-1} f=\chi(D) f ; \forall q \in \Delta_{q} f=\varphi\left(2^{-q} D\right) f$ and $\forall q \in \dot{\Delta}_{q} f=\varphi\left(2^{-q} D\right) f$.

The low-frequency cut-offs are denoted:

$$
\begin{gathered}
S_{q} f=\sum_{-1 \leq q^{\prime} \leq q-1} \Delta_{q^{\prime}} f, \\
\dot{S}_{q} f=\sum_{q^{\prime} \leq q-1} \dot{\Delta}_{q}^{\prime} f .
\end{gathered}
$$

Now, we introduce the definition of the Besov space. For $s \in \mathbb{R}, 1 \leq p, r \leq \infty$, the homogenous Besov space $\dot{B}_{p, r}^{s}$ is 
defined as the set of tempered distributions of $f \in \mathcal{S}^{\prime} / \mathscr{P}$ satisfying

$$
\|f\|_{\dot{B}_{p, r}^{s}}:=\left(\sum_{q \in Z} 2^{q s r}\left\|\dot{\Delta}_{q} f\right\|_{p}^{r}\right)^{1 / r}<\infty
$$

where $\mathscr{P}$ is the polynomial space. The inhomogeneous space $B_{p, r}^{s}$ is the set of tempered distribution $f$ with the norm

$$
\|f\|_{B_{p, r}^{s}}:=\left(\sum_{q \geq-1} 2^{q s r}\left\|\Delta_{q} f\right\|_{p}^{r}\right)^{1 / r}<\infty
$$

It is worthwhile to remark that $B_{2,2}^{s}$ and $B_{\infty, \infty}^{s}$ coincide with the usual Sobolev spaces $H^{s}$ and the usual Hölder space $C^{s}$ for $s \in \mathbb{R} \backslash \mathbb{Z}$, respectively.

In our study, we require the space-time Besov spaces as the following manner: for $T>0$ and $\rho \geq 1$, we denote by $L_{T}^{\rho}$ $B_{p, r}^{s}$ the set of all tempered distribution $f$ such that

$$
\|f\|_{L_{T}^{\rho} B_{p, r}^{s}} \triangleq\left\|\left(\sum_{q \in \mathbb{Z}} 2^{q s r}\left\|\dot{\Delta}_{q} f\right\|_{L^{p}\left(\mathbb{R}^{d}\right)}^{r}\right)^{1 / r}\right\|_{L_{T}^{\rho}}<\infty
$$

Lemma 3 (see [22]). Let $1 \leq p \leq q \leq \infty$. Suppose that $f \in L^{p}$, then there exists a constant $C$ independent of $f, j$ such that

$$
\begin{gathered}
\sup \hat{p f} \subset\left\{|\xi| \leq C 2^{j}\right\} \Rightarrow\left\|\partial^{\alpha} f\right\|_{q} \leq C 2^{j|\alpha|+d j(1 / p-1 / q)}\|f\|_{p}, \\
\sup p \widehat{f} \subset\left\{\frac{1}{C} 2^{j} \leq|\xi| \leq C 2^{j}\right\} \Rightarrow\|f\|_{p} \leq C 2^{-j|\alpha|} \sup _{|\beta|=|\alpha|}\left\|\partial^{\beta} f\right\|_{p} .
\end{gathered}
$$

Lemma 4 (see [22]). There exists a constant $C>0$ such that for $s>0$, we have

$$
\|u v\|_{H^{s}} \leq C\|u\|_{\infty}\|v\|_{H^{s}}+C\|u\|_{H^{s}}\|v\|_{\infty} .
$$

Lemma 5 (see [23]). Let $u$ be a solution of the transport equation

$$
\left\{\begin{array}{l}
u_{t}+v \cdot \nabla u=0 \\
u(x, 0)=u_{0}
\end{array}\right.
$$

and define $R_{q}:=v \cdot \nabla \Delta_{q} u-\Delta_{q}(v \cdot \nabla u), 1 \leq p \leq p_{1} \leq \infty, 1 \leq r$ $\leq \infty$, and $s \in$ such that $s>-d \min \left(1 / p_{1}, 1 / p^{\prime}\right)($ or $s>-1-d$ $\min \left(1 / p_{1}, 1 / p^{\prime}\right)$ if $\left.\operatorname{div} v=0\right)$. There exists a sequence $c_{q} \epsilon$ $\ell^{r}(\mathbb{Z})$ such that $\left\|c_{q}\right\|_{\ell^{r}}=1$ and a constant $C$ depending only on $d, r, s, p$, and $p_{1}$, which satisfy

$$
\forall q \in \mathbb{Z}, 2^{q s}\left\|R_{q}\right\|_{p} \leq C c_{q} Z^{\prime}(t)\|u\|_{B_{p, r}^{s}}
$$

with

$$
Z^{\prime}(t):=\left\{\begin{array}{l}
\|\nabla v\|_{B_{p_{1}, \infty}^{d / p_{1} \cap L^{\infty}}}, \text { if } s<1+\frac{d}{p_{1}}, \\
\|\nabla v\|_{B_{p_{1}, r}^{s-1}}, \text { if either } s>1+\frac{d}{p_{1}} \text { or } s=1+\frac{d}{p_{1}} \text { for } r=1 .
\end{array}\right.
$$

Lemma 6 (see [24]). Let $[p, r] \in[1, \infty]^{2}$, $v$ be a divergence-free vector-field belonging to the space $L_{\text {loc }}^{1}\left(\mathbb{R}_{+} ; \operatorname{Lip}\left(\mathbb{R}^{d}\right)\right.$ and let a be a smooth solution of the following transport equation:

$$
\left\{\begin{array}{l}
a_{t}+v \cdot \nabla a=f \\
a(x, 0)=a_{0}
\end{array}\right.
$$

If the initial data $a_{0} \in B_{p, r}^{0}$, then we have for all $t \in+\mathbb{R}$

$$
\|a\|_{\tilde{L}_{t}^{\infty} B_{p, r}^{0}} \leq C\left(\left\|a_{0}\right\|_{B_{p, r}^{0}}+\|f\|_{\tilde{L}_{t}^{1} B_{p, r}^{0}}\right)\left(1+\int_{0}^{t}\|\nabla v(\tau)\|_{L^{\infty}} d \tau\right) .
$$

\section{Proof of Theorems}

3.1. Local Well-Posedness. We construct the following smoothing system:

$$
\left\{\begin{array}{l}
n_{t}^{k}+u^{k} \cdot \nabla n^{k}=\Delta n^{k}-\nabla \cdot\left(n^{k} \nabla c^{k}\right)+n^{k}-\left(n^{k}\right)^{2}, \quad k \in N, \\
c_{t}^{k}+u^{k} \cdot \nabla c^{k}=\Delta c^{k}-c^{k} n^{k}, \\
u_{t}^{k}+\left(u^{k} \cdot \nabla\right) u^{k}-\nabla P^{k}=\Delta u^{k}+n^{k} \nabla \Phi, \\
\nabla \cdot u^{k}=0, \\
\left.\left(n^{k}, c^{k}, u^{k}\right)\right|_{t=0}=\left(S_{k} n^{0}, S_{k} c^{0}, S_{k} u^{0}\right) .
\end{array}\right.
$$

Step 1. Uniform boundedness.

Taking the operation $\Delta_{q}$ with $q \geq-1$ on the first equation of (25), we obtain

$$
\Delta_{q} n_{t}^{k}+\Delta_{q}\left(u^{k} \cdot \nabla n^{k}\right)=\Delta_{q} n^{k}-\nabla \cdot \Delta_{q}\left(n^{k} \nabla c^{k}\right)+\Delta_{q} n^{k}-\Delta_{q}\left(n^{k}\right)^{2} .
$$


Making the $L^{2}$-inner product for (26) with $\Delta_{q} n^{k}$ yields

$$
\begin{aligned}
& \frac{1}{2} \frac{d}{d t}\left\|\Delta_{q} n^{k}\right\|_{2}^{2}+\left\|\nabla \Delta_{q} n^{k}\right\|_{2}^{2}=-\int_{\mathbb{R}^{d}} \Delta_{q}\left(u^{k} \cdot \nabla n^{k}\right) \Delta_{q} n^{k} d x \\
& \quad-\int_{\mathbb{R}^{d}} \nabla \cdot \Delta_{q}\left(n^{k} \nabla c^{k}\right) \Delta_{q} n^{k} d x+\int_{\mathbb{R}^{d}} \Delta_{q} n^{k} \Delta_{q} n^{k} d x \\
& \quad-\int_{\mathbb{R}^{d}} \Delta_{q}\left(n^{k}\right)^{2} \Delta_{q} n^{k} d x \leq\left\|\Delta_{q}\left(u^{k} \cdot \nabla n^{k}\right)\right\|_{2}\left\|\Delta_{q} n^{k}\right\|_{2}+\| \Delta_{q} \\
& \cdot\left(n^{k} \nabla c^{k}\right)\left\|_{2}\right\| \nabla \Delta_{q} n^{k}\left\|_{2}+\right\| \Delta_{q} n^{k}\left\|_{2}^{2}+\right\| \Delta_{q}\left(n^{k}\right)^{2}\left\|_{2}\right\| \Delta_{q} n^{k} \|_{2} .
\end{aligned}
$$

Multiplying $2^{2 q s}$ on both sides of the above inequality, then taking the $\ell^{1}$ norm, using Hölder's inequality and Young's inequality together with Lemma 4, we have

$$
\begin{aligned}
& \frac{1}{2} \frac{d}{d t}\left\|n^{k}\right\|_{H^{s}}^{2}+\left\|n^{k}\right\|_{H^{s+1}}^{2} \leq\left\|u^{k} \cdot \nabla n^{k}\right\|_{H^{s}}\left\|n^{k}\right\|_{H^{s}}+\left\|n^{k} \nabla c^{k}\right\|_{H^{s}}\left\|n^{k}\right\|_{H^{s+1}} \\
&+\left\|n^{k}\right\|_{H^{s}}^{2}+\left\|\left(n^{k}\right)^{2}\right\|_{H^{s}}\left\|n^{k}\right\|_{H^{s}} \leq C\left\|u^{k}\right\|_{H^{s}}\left\|n^{k}\right\|_{H^{s+1}}\left\|n^{k}\right\|_{H^{s}} \\
&+ C\left\|n^{k}\right\|_{H^{s}}\left\|c^{k}\right\|_{H^{s+1}}\left\|n^{k}\right\|_{H^{s+1}}+\left\|n^{k}\right\|_{H^{s}}^{2}+C\left\|n^{k}\right\|_{H^{s}}^{2}\left\|n^{k}\right\|_{H^{s}} \\
& \leq C\left\|u^{k}\right\|_{H^{s}}^{2}\left\|n^{k}\right\|_{H^{s}}^{2}+\frac{1}{8}\left\|n^{k}\right\|_{H^{s+1}}^{2}+C\left\|n^{k}\right\|_{H^{s}}^{2}\left\|c^{k}\right\|_{H^{s+1}}^{2} \\
&+\frac{1}{8}\left\|n^{k}\right\|_{H^{s+1}}^{2}+\left\|n^{k}\right\|_{H^{s}}^{2}+C\left(\left\|n^{k}\right\|_{H^{s}}^{4}+\left\|n^{k}\right\|_{H^{s}}^{2}\right) .
\end{aligned}
$$

Then, we conclude

$\frac{d}{d t}\left\|n^{k}\right\|_{H^{s}}^{2}+\left\|n^{k}\right\|_{H^{s+1}}^{2} \leq C\left(\left\|u^{k}\right\|_{H^{s}}^{2}\left\|n^{k}\right\|_{H^{s}}^{2}+\left\|n^{k}\right\|_{H^{s}}^{2}\left\|c^{k}\right\|_{H^{s+1}}^{2}+\left\|n^{k}\right\|_{H^{s}}^{2}+\left\|n^{k}\right\|_{H^{s}}^{4}\right)$.

In a similar way to (29), we obtain

$$
\begin{aligned}
\frac{1}{2} \frac{d}{d t}\left\|c^{k}\right\|_{H^{s+1}}^{2} & +\left\|c^{k}\right\|_{H^{s+2}}^{2} \leq C\left\|u^{k}\right\|_{H^{s+1}}^{2}\left\|c^{k}\right\|_{H^{s+1}}^{2} \\
& +\frac{1}{8}\left\|c^{k}\right\|_{H^{s+2}}^{2}+C\left\|c^{k}\right\|_{H^{s+1}}^{4}+\frac{1}{8}\left\|n^{k}\right\|_{H^{s+1}}^{2}
\end{aligned}
$$

Thus, we have

$$
\frac{d}{d t}\left\|c^{k}\right\|_{H^{s+1}}^{2}+\left\|c^{k}\right\|_{H^{s+2}}^{2} \leq C\left(\left\|u^{k}\right\|_{H^{s+1}}^{2}\left\|c^{k}\right\|_{H^{s+1}}^{2}+\left\|c^{k}\right\|_{H^{s+1}}^{4}\right)+\frac{1}{8}\left\|n^{k}\right\|_{H^{s+1}}^{2} .
$$

Operating $\Delta_{q}$ with $q \geq-1$ to the third equation of (25) implies

$$
\begin{aligned}
\Delta_{q} u_{t}^{k} & +\left(u^{k} \cdot \nabla\right) \Delta_{q} u^{k}-\nabla \Delta_{q} P^{k}=\left(u^{k} \cdot \nabla\right) \Delta_{q} u^{k} \\
& -\Delta_{q}\left(\left(u^{k} \cdot \nabla\right) u^{k}\right)-\Delta_{q}\left(n^{k} \nabla \Phi\right) .
\end{aligned}
$$

Taking the $L^{2}$-inner product for the above equality with
$\Delta_{q} u^{k}$ gives

$$
\begin{aligned}
\frac{1}{2} \frac{d}{d t}\left\|\Delta_{q} u^{k}\right\|_{2}^{2}= & \int_{\mathbb{R}^{d}}\left(\left(u^{k} \cdot \nabla\right) \Delta_{q} u^{k}-\Delta_{q}\left(\left(u^{k} \cdot \nabla\right) u^{k}\right)\right) \Delta_{q} u^{k} d x \\
& -\int_{\mathbb{R}^{d}} \Delta_{q}\left(n^{k} \nabla \Phi\right) \Delta_{q} u^{k} d x \leq \|\left(u^{k} \cdot \nabla\right) \Delta_{q} u^{k} \\
& -\Delta_{q}\left(\left(u^{k} \cdot \nabla\right) u^{k}\right)\left\|_{2}\right\| \Delta_{q} u^{k}\left\|_{2}+\right\| \Delta_{q}\left(n^{k} \nabla \Phi\right)\left\|_{2}\right\| \Delta_{q} u^{k} \|_{2} .
\end{aligned}
$$

Multiplying $2^{2 q(s+1)}$ on both sides of the above inequality and taking the $\ell^{1}$ norm, we conclude

$$
\begin{aligned}
\frac{d}{d t}\left\|u^{k}\right\|_{H^{s+1}}^{2} & \leq\left\|\nabla u^{k}\right\|_{\infty}\left\|u^{k}\right\|_{H^{s+1}}^{2}+\frac{1}{8}\left\|n^{k}\right\|_{H^{s+1}}^{2}+\left\|u^{k}\right\|_{H^{s+1}}^{2} \\
& \leq C\left(\left\|u^{k}\right\|_{H^{s+1}}^{4}+\left\|u^{k}\right\|_{H^{s+1}}^{2}\right)+\frac{1}{8}\left\|n^{k}\right\|_{H^{s+1}}^{2} .
\end{aligned}
$$

Collecting (29)-(34), we have

$$
\begin{aligned}
& \frac{d}{d t}\left(\left\|n^{k}\right\|_{H^{s}}^{2}+\left\|c^{k}\right\|_{H^{s+1}}^{2}+\left\|u^{k}\right\|_{H^{s+1}}^{2}\right)+\left\|n^{k}\right\|_{H^{s+1}}^{2}+\left\|c^{k}\right\|_{H^{s+2}}^{2} \\
& \quad \leq C\left(\left\|n^{k}\right\|_{H^{s}}^{2}+\left\|c^{k}\right\|_{H^{s+1}}^{2}+\left\|u^{k}\right\|_{H^{s+1}}^{2}\right)\left(1+\left\|n^{k}\right\|_{H^{s}}^{2}+\left\|c^{k}\right\|_{H^{s+1}}^{2}+\left\|u^{k}\right\|_{H^{s+1}}^{2}\right) \\
& \quad \leq\left(1+\left\|n^{k}\right\|_{H^{s}}^{2}+\left\|c^{k}\right\|_{H^{s+1}}^{2}+\left\|u^{k}\right\|_{H^{s+1}}^{2}\right)^{2} .
\end{aligned}
$$

We obtain from the Gronwall inequality that

$$
1+\left\|n^{k}\right\|_{H^{s}}^{2}+\left\|c^{k}\right\|_{H^{s+1}}^{2}+\left\|u^{k}\right\|_{H^{s+1}}^{2} \leq \frac{1+\left\|n_{0}^{k}\right\|_{H^{s}}^{2}+\left\|c_{0}^{k}\right\|_{H^{s+1}}^{2}+\left\|u_{0}^{k}\right\|_{H^{s+1}}^{2}}{1-C\left(1+\left\|n_{0}^{k}\right\|_{H^{s}}^{2}+\left\|c_{0}^{k}\right\|_{H^{s+1}}^{2}+\left\|u_{0}^{k}\right\|_{H^{s+1}}^{2}\right) t} .
$$

Let

$$
T=\frac{1}{2 C\left(1+\left\|n_{0}^{k}\right\|_{H^{s}}^{2}+\left\|c_{0}^{k}\right\|_{H^{s+1}}^{2}+\left\|u_{0}^{k}\right\|_{H^{s+1}}^{2}\right)}>0,
$$

then we obtain

$$
\begin{aligned}
& \sup _{t \in[0, T]}\left(\left\|n^{k}(t)\right\|_{H^{s}}^{2}+\left\|c^{k}(t)\right\|_{H^{s+1}}^{2}+\left\|u^{k}(t)\right\|_{H^{s+1}}^{2}\right) \\
& +\int_{0}^{t}\left(\left\|n^{k}\right\|_{H^{s+1}}^{2}+\left\|c^{k}\right\|_{H^{s+2}}^{2}\right)(\tau) d \tau \\
& \quad \leq 2\left(1+\left\|n_{0}^{k}\right\|_{H^{s}}^{2}+\left\|c_{0}^{k}\right\|_{H^{s+1}}^{2}+\left\|u_{0}^{k}\right\|_{H^{s+1}}^{2}\right) .
\end{aligned}
$$

Step 2. Extracting sequences.

According to (38), we get

$$
\begin{gathered}
n^{k} \in L^{\infty}\left([0, T], H^{s}\right) \cap L^{2}\left([0, T], H^{s+1}\right), \\
c^{k} \in L^{\infty}\left([0, T], H^{s+1}\right) \cap L^{2}\left([0, T], H^{s+2}\right), \\
u^{k} \in L^{\infty}\left([0, T], H^{s+1}\right) .
\end{gathered}
$$


In order to prove the convergence, we require uniform boundedness for $\partial_{t} n^{k}, \partial_{t} c^{k}$, and $\partial_{t} u^{k}$. By the first equation of (25), we infer

$$
\begin{aligned}
\left\|\partial_{t} n^{k}\right\|_{L_{t}^{\infty} H^{-1}} \leq & \left\|\Delta n^{k}\right\|_{L_{t}^{\infty} H^{-1}}+\left\|u^{k} \cdot \nabla n^{k}\right\|_{L_{t}^{\infty} H^{-1}}+\| \nabla \\
& \cdot\left(n^{k} \nabla c^{k}\right)\left\|_{L_{t}^{\infty} H^{-1}}+\right\| n^{k}\left\|_{L_{t}^{\infty} H^{-1}}+\right\|\left(n^{k}\right)^{2} \|_{L_{t}^{\infty} H^{-1}} \\
\leq & \left\|n^{k}\right\|_{L_{t}^{\infty} H^{s}}+\left\|u^{k}\right\|_{L_{t}^{\infty} H^{s+1}}\left\|n^{k}\right\|_{L_{t}^{\infty} H^{s}}+\left\|n^{k}\right\|_{L_{t}^{\infty} H^{s}}\left\|c^{k}\right\|_{L_{t}^{\infty} H^{s+1}} \\
& +\left\|n^{k}\right\|_{L_{t}^{\infty} H^{s}}+\left\|n^{k}\right\|_{L_{t}^{\infty}}^{2} H^{s} \leq C .
\end{aligned}
$$

In a similar process, we have

$$
\begin{aligned}
\left\|\partial_{t} c^{k}\right\|_{L_{t}^{\infty} H^{-1}} \leq & \left\|\Delta c^{k}\right\|_{L_{t}^{\infty} H^{-1}}+\left\|u^{k} \cdot \nabla c^{k}\right\|_{L_{t}^{\infty} H^{-1}}+\left\|c^{k} n^{k}\right\|_{L_{t}^{\infty} H^{-1}} \\
\leq & \left\|c^{k}\right\|_{L_{t}^{\infty} H^{s+1}}+\left\|u^{k}\right\|_{L_{t}^{\infty} H^{s+1}}\left\|c^{k}\right\|_{L_{t}^{\infty} H^{s+1}} \\
& +\left\|c^{k}\right\|_{L_{t}^{\infty} H^{s+1}}\left\|n^{k}\right\|_{L_{t}^{\infty} H^{s}} \leq C . \\
\left\|\partial_{t} u^{k}\right\|_{L_{t}^{\infty} H^{-1}} \leq & \left\|\left(u^{k} \cdot \nabla\right) u^{k}\right\|_{L_{t}^{\infty} H^{-1}}+\left\|n^{k} \nabla \Phi\right\|_{L_{t}^{\infty} H^{-1}} \\
\leq & \left\|u^{k}\right\|_{L_{t}^{\infty} H^{s+1}}^{2}+\left\|n^{k}\right\|_{L_{t}^{\infty} H^{s}} \leq C .
\end{aligned}
$$

Since $L^{2}$ is locally compactly embedded in $H^{-1}$, we can apply the Aubin-Lions Lemma to deduce that, extracting a subsequence, the approximate solution sequence $\left(n^{k}, c^{k}, u^{k}\right)$ strongly converges in $\mathrm{L}^{\infty}\left([0, T] ; H^{-1}\right)$ to some function $(n$, $c, u)$ such that

$$
\begin{gathered}
n^{k} \in L^{\infty}\left([0, T] ; H^{s}\right) \cap L^{2}\left([0, T], H^{s+1}\right), \\
c^{k} \in L^{\infty}\left([0, T] ; H^{s+1}\right) \cap L^{2}\left([0, T], H^{s+2}\right), \\
u^{k} \in L^{\infty}\left([0, T] ; H^{s+1}\right) .
\end{gathered}
$$

By the above estimates, we can easily have the limit in the approximate system (25) and $(n, c, u)$ solve (3) in the sense of distribution. Using a classical method [12], we have $n \in C([$ $\left.0, T] ; H^{s}\right), c \in C\left([0, T] ; H^{s+1}\right)$, and $u \in C\left([0, T] ; H^{s+1}\right)$.

\section{Step 3. Uniqueness.}

Let us consider the two solutions $\left(n_{1}, c_{1}, u_{1}\right)$ and $\left(n_{2}, c_{2}\right.$ ,$u_{2}$ ) associated with the same initial data and satisfy (3). We use the notation $\delta n=n_{1}-n_{2}, \delta c=c_{1}-c_{2}$, and $\delta u=u_{1}$ $-u_{2}$. Then, we have

$$
\left\{\begin{array}{l}
\partial_{t} \delta n+\delta u \cdot \nabla n_{1}+u_{2} \cdot \nabla \delta n=\Delta \delta n-\nabla \cdot\left(\delta n \nabla c_{1}\right)-\nabla \cdot\left(n_{2} \nabla \delta c\right)+\delta n-n_{1} \delta n-n_{2} \delta n, \\
\partial_{t} \delta c+\delta u \cdot \nabla c_{1}+u_{2} \cdot \nabla \delta c=\Delta \delta c-n_{1} \delta c-c_{2} \delta n \\
\partial_{t} \delta u+(\delta u \cdot \nabla) u_{1}+\left(u_{2} \cdot \nabla\right) \delta u-\nabla\left(P_{1}-P_{2}\right)=\Delta \delta u+\delta n \nabla \Phi .
\end{array}\right.
$$

Multiplying the first equation of (43) by $\delta n$ and integrat- ing in spaces, we obtain

$$
\begin{aligned}
\frac{1}{2} \frac{d}{d t}\|\delta n(t)\|_{2}^{2}+\|\nabla \delta n(t)\|_{2}^{2}=-\int_{\mathbb{R}^{d}}\left(\delta u \cdot \nabla n_{1}\right) \delta n d x \\
\quad-\int_{\mathbb{R}^{d}} \nabla \cdot\left(\delta u \nabla c_{1}\right) \delta n d x-\int_{\mathbb{R}^{d}} \nabla \cdot\left(n_{2} \nabla \delta c\right) \delta n d x \\
+\int_{\mathbb{R}^{d}} \delta n \delta n d x-\int_{\mathbb{R}^{d}} n_{1} \delta n \delta n d x-\int_{\mathbb{R}^{d}} n_{2} \delta n \delta n d x \\
\quad \leq C\left(\|\delta u\|_{2}^{2}+\|\delta n\|_{2}^{2}\left\|n_{1}\right\|_{H^{s}}^{2}\right)+C\|\delta n\|_{2}^{2}\left\|c_{1}\right\|_{H^{s+1}}^{2} \\
+\frac{1}{8}\|\nabla \delta n\|_{2}^{2}+C\left\|n_{2}\right\|_{H^{s}}^{2}\|\nabla \delta c\|_{2}^{2}+\frac{1}{8}\|\nabla \delta n\|_{2}^{2}+\|\delta n\|_{2}^{2} \\
+\left\|n_{1}\right\|_{H^{s}}\|\delta n\|_{2}^{2}+\left\|n_{2}\right\|_{H^{s}}\|\delta n\|_{2}^{2},
\end{aligned}
$$

from which we conclude

$$
\begin{aligned}
\frac{d}{d t}\|\delta n(t)\|_{2}^{2} & +\|\nabla \delta n(t)\|_{2}^{2} \leq C\left(\|\delta u\|_{2}^{2}+\|\delta n\|_{2}^{2}\left\|n_{1}\right\|_{H^{s}}^{2}\right. \\
& +\|\delta n\|_{2}^{2}\left\|c_{1}\right\|_{H^{s+1}}^{2}+\left\|n_{2}\right\|_{H^{s}}^{2}\|\nabla \delta c\|_{2}^{2}+\|\delta n\|_{2}^{2} \\
& \left.+\left\|n_{1}\right\|_{H^{s}}\|\delta n\|_{2}^{2}+\left\|n_{2}\right\|_{H^{s}}\|\delta n\|_{2}^{2}\right) .
\end{aligned}
$$

Then, multiplying the second equation of (43) by $\delta c$ and integrating in spaces, we know

$$
\begin{gathered}
\frac{1}{2} \frac{d}{d t}\|\delta c(t)\|_{2}^{2}+\|\nabla \delta c(t)\|_{2}^{2}=-\int_{\mathbb{R}^{d}}\left(\delta u \cdot \nabla c_{1}\right) \delta c d x \\
-\int_{\mathbb{R}^{d}} \delta c n_{1} \delta c d x-\int_{\mathbb{R}^{d}} \delta n c_{2} \delta c d x \\
\leq C\left(\|\delta u\|_{2}^{2}+\|\delta c\|_{2}^{2}\left\|c_{1}\right\|_{H^{s+1}}^{2}+\|\delta c\|_{2}^{2}\left\|n_{1}\right\|_{H^{s}}\right. \\
\left.+\|\delta n\|_{2}^{2}+\|\delta c\|_{2}^{2}\left\|c_{2}\right\|_{H^{s+1}}^{2}\right) .
\end{gathered}
$$

Hence, we get

$$
\begin{gathered}
\frac{d}{d t}\|\delta c(t)\|_{2}^{2}+\|\nabla \delta c(t)\|_{2}^{2} \leq C\left(\|\delta u\|_{2}^{2}+\|\delta c\|_{2}^{2}\left\|c_{1}\right\|_{H^{s+1}}^{2}+\|\delta c\|_{2}^{2}\left\|n_{1}\right\|_{H^{s}}\right. \\
\left.+\|\delta n\|_{2}^{2}+\|\delta c\|_{2}^{2}\left\|c_{2}\right\|_{H^{s+1}}^{2}\right) .
\end{gathered}
$$

Applying $\partial_{i}$ on both sides of the second equation of Equation (43) gives

$$
\begin{aligned}
\partial_{t} \partial_{i} \delta c & +u_{2} \cdot \nabla \partial_{i} \nabla c-\Delta \partial_{i} \delta c=-\partial_{i}\left(\delta u \cdot \nabla c_{1}\right)-\partial_{i} u_{2} \cdot \nabla \delta c \\
& -\partial_{i}\left(n_{1} \delta c\right)-\partial_{i}\left(c_{2} \delta n\right) .
\end{aligned}
$$

Taking the $L^{2}$-inner product for the above equation with 
$\partial_{i} \delta c$, we obtain

$$
\begin{aligned}
\frac{1}{2} \frac{d}{d t}\|\nabla \delta c(t)\|_{2}^{2} & +\|\Delta \delta c(t)\|_{2}^{2}=-\sum_{i} \int_{\mathbb{R}^{d}} \partial_{i}\left(\delta u \cdot \nabla c_{1}\right) \partial_{i} \delta c d x \\
& -\sum_{i} \int_{\mathbb{R}^{d}} \partial_{i} u_{2} \cdot \nabla \delta c \partial_{i} \delta c d x \\
& -\sum_{i} \int_{\mathbb{R}^{d}} \partial_{i}\left(n_{1} \delta c\right) \partial_{i} \delta c d x \\
& -\sum_{i} \int_{\mathbb{R}^{d}} \partial_{i}\left(c_{2} \delta n\right) \partial_{i} \delta c d x=\int_{\mathbb{R}^{d}}\left(\delta u \cdot \nabla c_{1}\right) \Delta \delta c d x \\
& -\int_{\mathbb{R}^{d}}(\nabla \delta c \cdot \nabla) u_{2} \cdot \nabla \delta c d x+\int_{\mathbb{R}^{d}} n_{1} \delta c \Delta \delta c d x \\
& +\int_{\mathbb{R}^{d}} c_{2} \delta n \Delta \delta c d x \leq C\|\delta u\|_{2}^{2}\left\|c_{1}\right\|_{H^{s+1}}^{2}+\frac{1}{8}\|\Delta \delta c\|_{2}^{2} \\
& +C\|\nabla \delta c\|_{2}^{2}\left\|u_{2}\right\|_{H^{s+1}}+C\|\delta c\|_{2}^{2}\left\|n_{1}\right\|_{H^{s}}^{2}+\frac{1}{8}\|\Delta \delta c\|_{2}^{2} \\
& +C\|\delta n\|_{2}^{2} \mid c_{2}\left\|_{H^{s+1}}^{2}+\frac{1}{8}\right\| \Delta \delta c \|_{2}^{2} .
\end{aligned}
$$

Hence, we have

$$
\begin{gathered}
\frac{d}{d t}\|\nabla \delta c(t)\|_{2}^{2}+\|\Delta \delta c(t)\|_{2}^{2} \leq C\left(\|\delta u\|_{2}^{2}\left\|c_{1}\right\|_{H^{s+1}}^{2}+\|\nabla \delta c\|_{2}^{2}\left\|u_{2}\right\|_{H^{s+1}}\right. \\
\left.+\|\delta c\|_{2}^{2}\left\|n_{1}\right\|_{H^{s}}^{2}+\|\delta n\|_{2}^{2} \mid c_{2} \|_{H^{s+1}}^{2}\right)
\end{gathered}
$$

Multiplying the third equation of system (43) by $\delta u$ and integrating in spaces, we get

$$
\begin{aligned}
& \frac{1}{2} \frac{d}{d t}\|\delta u(t)\|_{2}^{2}=-\int_{\mathbb{R}^{d}}\left((\delta u \cdot \nabla) u_{1}\right) \cdot \delta u d x+\int_{\mathbb{R}^{d}} \delta n \nabla \Phi \cdot \delta u d x \\
& \quad \leq C\|\delta u\|_{2}^{2}\left\|u_{1}\right\|_{H^{s+1}}+C\left(\|\delta n\|_{2}^{2}+\|\delta u\|_{2}^{2}\right) .
\end{aligned}
$$

Thus,

$$
\frac{d}{d t}\|\delta u(t)\|_{2}^{2} \leq C\left(\|\delta u\|_{2}^{2}\left\|u_{1}\right\|_{H^{s+1}}+\|\delta n\|_{2}^{2}+\|\delta u\|_{2}^{2}\right)
$$

From (45)-(52), we obtain

$$
\begin{aligned}
& \frac{d}{d t}\left(\|\delta n(t)\|_{2}^{2}+\|\delta c(t)\|_{2}^{2}+\|\nabla \delta c(t)\|_{2}^{2}+\|\delta u(t)\|_{2}^{2}\right)+\|\nabla \delta n\|_{2}^{2} \\
& \quad+\|\nabla \delta c\|_{2}^{2}+\|\Delta \delta c\|_{2}^{2} \leq C\left(\|\delta u\|_{2}^{2}+\|\delta n\|_{2}^{2}\left\|n_{1}\right\|_{H^{s}}^{2}+\|\delta n\|_{2}^{2}\left\|c_{1}\right\|_{H^{s+1}}^{2}\right. \\
& \quad+\left\|n_{2}\right\|_{H^{s}}^{2}\|\nabla \delta c\|_{2}^{2}+\|\delta n\|_{2}^{2}+\left\|n_{1}\right\|_{H^{s}}\|\delta n\|_{2}^{2}+\left\|n_{2}\right\|_{H^{s}}\|\delta n\|_{2}^{2}+\|\delta u\|_{2}^{2} \\
& \quad+\|\delta c\|_{2}^{2}\left\|c_{1}\right\|_{H^{s+1}}^{2}+\|\delta c\|_{2}^{2}\left\|n_{1}\right\|_{H^{s}}+\|\delta n\|_{2}^{2}+\|\delta c\|_{2}^{2}\left\|c_{2}\right\|_{H^{s+1}}^{2} \\
& \quad+\|\delta u\|_{2}^{2}\left\|c_{1}\right\|_{H^{s+1}}^{2}+\|\nabla \delta c\|_{2}^{2}\left\|u_{2}\right\|_{H^{s+1}}+\|\delta c\|_{2}^{2}\left\|n_{1}\right\|_{H^{s}}^{2} \\
& \left.\quad+\|\delta n\|_{2}^{2} \mid c_{2}\left\|_{H^{s+1}}^{2}+\right\| \delta u\left\|_{2}^{2}\right\| u_{1}\left\|_{H^{s+1}}+\right\| \delta n\left\|_{2}^{2}+\right\| \delta u \|_{2}^{2}\right) .
\end{aligned}
$$

Then, we have

$$
\begin{gathered}
\frac{d}{d t}\left(\|\delta n(t)\|_{2}^{2}+\|\delta c(t)\|_{2}^{2}+\|\nabla \delta c(t)\|_{2}^{2}+\|\delta u(t)\|_{2}^{2}\right) \\
\leq C F(t)\left(\|\delta n\|_{2}^{2}+\|\delta c\|_{2}^{2}+\|\nabla \delta c\|_{2}^{2}+\|\delta u\|_{2}^{2}\right),
\end{gathered}
$$

where

$$
\begin{aligned}
F(t)= & 1+\left\|n_{1}\right\|_{H^{s}}+\left\|n_{2}\right\|_{H^{s}}+\left\|u_{1}\right\|_{H^{s+1}}+\left\|u_{2}\right\|_{H^{s+1}}+\left\|n_{1}\right\|_{H^{s}}^{2}+\left\|n_{2}\right\|_{H^{s}}^{2} \\
& +\left\|c_{1}\right\|_{H^{s+1}}^{2}+\left\|c_{2}\right\|_{H^{s+1}}^{2} .
\end{aligned}
$$

From (3), we infer that $F(t)$ is integrable. Using the Gronwall inequality gives the uniqueness.

3.2. Blowup Criterion. Operating $\Delta_{q}$ with $q \geq-1$ to the first equation of (3) gives

$$
\begin{aligned}
\Delta_{q} n_{t} & +u \cdot \nabla \Delta_{q} n=\Delta_{q} n+u \cdot \nabla \Delta_{q} n-\Delta_{q}(u \cdot \nabla n)-\nabla \cdot \Delta_{q}(n \nabla c) \\
& +\Delta_{q} n-\Delta_{q} n^{2} .
\end{aligned}
$$

Taking the $L^{2}$-inner product for the above equation with $\Delta_{q} n$, we get

$$
\begin{aligned}
\frac{1}{2} \frac{d}{d t}\left\|\Delta_{q} n\right\|_{2}^{2} & +\nabla \Delta_{q} n \|_{2}^{2}=\int_{d}\left(u \cdot \nabla \Delta_{q} n-\Delta_{q}(u \cdot \nabla n)\right) \Delta_{q} n d x \\
& +\int_{d} \Delta_{q}(n \nabla c) \cdot \nabla \Delta_{q} n d x+\int_{d} \Delta_{q} n \Delta_{q} n d x \\
& -\int_{d} \Delta_{q} n^{2} \Delta_{q} n d x \leq\left\|u \cdot \nabla \Delta_{q} n-\Delta_{q}(u \cdot \nabla n)\right\|_{2}\left\|\Delta_{q} n\right\|_{2} \\
& +\left\|\Delta_{q}(n \nabla c)\right\|_{2}\left\|\nabla \Delta_{q} n\right\|_{2}+\left\|\Delta_{q} n\right\|_{2}^{2}+\left\|\Delta_{q} n^{2}\right\|_{2}\left\|\Delta_{q} n\right\|_{2} .
\end{aligned}
$$

Multiplying $2^{2 q s}$ on both sides of the above inequality and performing $\ell_{1}$ norm, we have

$$
\begin{gathered}
\frac{d}{d t}\|n\|_{H^{s}}^{2}+\|n\|_{H^{s+1}}^{2} \leq\|\nabla u\|_{\infty}\|n\|_{H^{s}}^{2}+\|n \nabla c\|_{H^{s}}\|n\|_{H^{s+1}}+\|n\|_{H^{s}}^{2} \\
+\left\|n^{2}\right\|_{H^{s}}\|n\|_{H^{s}} .
\end{gathered}
$$

Using Young's inequality, we conclude

$$
\begin{aligned}
\frac{d}{d t}\|n\|_{H^{s}}^{2} & \|n\|_{H^{s+1}}^{2} \leq C\|\nabla u\|_{\infty}\|n\|_{H^{s}}^{2}+C\|n\|_{\infty}^{2}\|\nabla c\|_{H^{s}}^{2} \\
& +C\|n\|_{H^{s}}^{2}\|\nabla c\|_{\infty}^{2}+\frac{1}{8}\|n\|_{H^{s+1}}^{2}+\|n\|_{H^{s}}^{2}+C\|n\|_{\infty}^{2}\|n\|_{H^{s}}^{2} \\
& +\frac{1}{8}\|n\|_{H^{s+1}}^{2} .
\end{aligned}
$$


In terms of the second equation of (3), we know

$$
\Delta_{q} c_{t}+u \cdot \nabla \Delta_{q} c=\Delta \Delta_{q} c+u \cdot \nabla \Delta_{q} c-\Delta_{q}(u \cdot \nabla c)-\Delta_{q}(n c) .
$$

Multiplying the above equality by $\Delta_{q} c$ and integrating in spaces mean

$$
\begin{aligned}
\frac{1}{2} \frac{d}{d t}\left\|\Delta_{q} c\right\|_{2}^{2} & +\left\|\nabla \Delta_{q} c\right\|_{2}^{2}=\int_{\mathbb{R}^{d}}\left(u \cdot \nabla \Delta_{q} c-\Delta_{q}(u \cdot \nabla c)\right) \Delta_{q} c d x \\
& +\int_{\mathbb{R}^{d}} \Delta_{q}(n c) \Delta_{q} c d x \leq\left\|u \cdot \nabla \Delta_{q} c-\Delta_{q}(u \cdot \nabla c)\right\|_{2}\left\|\Delta_{q} c\right\|_{2} \\
& +\left\|\Delta_{q}(n c)\right\|_{2}\left\|\Delta_{q} c\right\|_{2} .
\end{aligned}
$$

Multiplying $2^{2 q(s+1)}$ on both sides of the above inequality and taking $\ell_{1}$ norm, we obtain

$$
\frac{d}{d t}\|c\|_{H^{s+1}}^{2}+\|c\|_{H^{s+2}}^{2} \leq\|\nabla u\|_{\infty}\|c\|_{H^{s+1}}^{2}+\|n c\|_{H^{s+1}}\|c\|_{H^{s+1}}
$$

Utilizing Young's inequality, we have

$$
\begin{gathered}
\frac{d}{d t}\|c\|_{H^{s+1}}^{2}+\|c\|_{H^{s+2}}^{2} \leq C\|\nabla u\|_{\infty}\|c\|_{H^{s+1}}^{2}+C\|n\|_{\infty}^{2}\|c\|_{H^{s+1}}^{2} \\
+C\|c\|_{\infty}^{2}\|c\|_{H^{s+1}}^{2}+\frac{1}{8}\|n\|_{H^{s+1}}^{2} .
\end{gathered}
$$

According to the third equation of (3), we get

$\Delta_{q} u_{t}+(u \cdot \nabla) \Delta_{q} u-\nabla \Delta_{q} P=(u \cdot \nabla) \Delta_{q} u-\Delta_{q}((u \cdot \nabla) u)-\Delta_{q}(n \nabla \Phi)$.

Taking the $L^{2}$-inner product for the above equality with $\Delta_{q} u$ implies

$$
\begin{aligned}
\frac{1}{2} \frac{d}{d t}\left\|\Delta_{q} u\right\|_{2}^{2}= & \int_{\mathbb{R}^{d}}\left((u \cdot \nabla) \Delta_{q} u-\Delta_{q}((u \cdot \nabla) u)\right) \Delta_{q} u d x \\
& -\int_{\mathbb{R}^{d}} \Delta_{q}(n \nabla \Phi) \Delta_{q} u d x \leq \|(u \cdot \nabla) \Delta_{q} u \\
& -\Delta_{q}((u \cdot \nabla) u)\left\|_{2}\right\| \Delta_{q} u\left\|_{2}+\right\| \Delta_{q}(n \nabla \Phi)\left\|_{2}\right\| \Delta_{q} u \|_{2} .
\end{aligned}
$$

Multiplying $2^{2 q(s+1)}$ on both sides of the above inequality and taking the $\ell_{1}$ norm, we have

$$
\frac{d}{d t}\|u\|_{H^{s+1}}^{2} \leq C\|\nabla u\|_{\infty}\|u\|_{H^{s+1}}^{2}+\frac{1}{8}\|n\|_{H^{s+1}}^{2}+C\|u\|_{H^{s+1}}^{2} .
$$

Collecting (59)-(66), we deduce

$$
\begin{aligned}
& \frac{d}{d t}\left(\|n\|_{H^{s}}^{2}+\|c\|_{H^{s+1}}^{2}+\|u\|_{H^{s+1}}^{2}\right)+\|n\|_{H^{s+1}}^{2}+\|c\|_{H^{s+2}}^{2} \\
& \quad \leq\left(\|n\|_{H^{s}}^{2}+\|c\|_{H^{s+1}}^{2}+\|u\|_{H^{s+1}}^{2}\right)\left(1+\|\nabla u\|_{\infty}+\|n\|_{\infty}^{2}+\|\nabla c\|_{\infty}^{2}\right) .
\end{aligned}
$$

The Gronwall inequality implies

$$
\begin{aligned}
& \left(\|n(t)\|_{H^{s}}^{2}+\|c(t)\|_{H^{s+1}}^{2}+\|u(t)\|_{H^{s+1}}^{2}\right) \\
& \quad+\int_{0}^{t}\left(\|n(\tau)\|_{H^{s+1}}^{2}+\|c(\tau)\|_{H^{s+2}}^{2}\right) d \tau \\
& \quad \leq C \exp \left(\int_{0}^{t}\left(1+\|\nabla u(\tau)\|_{\infty}+\|n(\tau)\|_{\infty}^{2}+\|\nabla c(\tau)\|_{\infty}^{2}\right) d \tau\right) .
\end{aligned}
$$

Next, we turn to prove condition (11). Applying $\nabla$ on both sides of the second equation of (3) means

$$
\partial_{t} \nabla c+u \cdot \nabla^{2} c-\Delta \nabla c=-\nabla(n c)-\nabla u \cdot \nabla c .
$$

Multiplying the above equality with $|\nabla c|^{p-2} \nabla c$, we obtain

$$
\begin{aligned}
& \frac{1}{p} \frac{d}{d t}\|\nabla c\|_{p}^{p}+\int_{\mathbb{R}^{d}} u \cdot \nabla^{2} c|\nabla c|^{p-2} \nabla c d x+\frac{4(p-1)}{p^{2}}\left\|\nabla|\nabla c|^{p / 2}\right\|_{2}^{2}= \\
& -\int_{\mathbb{R}^{d}} \nabla(n c)|\nabla c|^{p-2} \nabla c d x-\int_{\mathbb{R}^{d}} \nabla u \cdot \nabla c|\nabla c|^{p-2} \nabla c d x \\
& \quad \leq(p-1) \int_{\mathbb{R}^{d}} n c|\nabla c|^{p-2} \Delta c d x+\|\nabla u\|_{\infty}\|\nabla c\|_{p}^{p} \\
& \quad \leq \frac{2(p-1)}{p}\|n\|_{\infty}\|c\|_{p}\left\||\nabla c|^{(p-2) / 2}\right\|_{2 p /(p-2)}\left\|\nabla|\nabla c|^{p / 2}\right\|_{2}+\|\nabla u\|_{\infty}\|\nabla c\|_{p}^{p} \\
& \quad \leq \frac{2(p-1)}{p}\left(\frac{p}{2(p-1) \varepsilon}\|n\|_{\infty}^{2}\|c\|_{p}^{2}\||\nabla c|\|_{p-2}^{2}+\frac{2(p-1) \varepsilon}{p}\left\|\nabla|\nabla c|^{p / 2}\right\|_{2}^{2}\right) \\
& \quad+\|\nabla u\|_{\infty}\|\nabla c\|_{p}^{p} .
\end{aligned}
$$

Because of

$$
\int_{\mathbb{R}^{d}} u \cdot \nabla^{2} c|\nabla c|^{p-2} \nabla c d x=\frac{1}{p} \int_{\mathbb{R}^{d}} u \cdot \nabla|\nabla c|^{p} d x=0,
$$

we get

$$
\frac{d}{d t}\|\nabla c\|_{p}^{2} \leq C\|n\|_{\infty}^{2}+\|\nabla u\|_{\infty}\|\nabla c\|_{p}^{2}
$$

Utilizing Gronwall's inequality, we have

$$
\begin{aligned}
\|\nabla c(t)\|_{p}^{2} & \leq C\left(\left\|\nabla c_{0}\right\|_{p}+\int_{0}^{t}\|n(\tau)\|_{\infty}^{2} d \tau\right) \exp \int_{0}^{t}\|\nabla u(\tau)\|_{\infty} d \tau \\
& \leq C\left(\left\|c_{0}\right\|_{H^{s}}+\int_{0}^{t}\|n(\tau)\|_{\infty}^{2} d \tau\right) \exp \int_{0}^{t}\|\nabla u(\tau)\|_{\infty} d \tau .
\end{aligned}
$$


Setting $p \rightarrow \infty$, we conclude

$$
\|\nabla c(t)\|_{\infty}^{2} \leq\left(\left\|c_{0}\right\|_{H^{s}}+\int_{0}^{t}\|n(\tau)\|_{\infty}^{2} d \tau\right) \exp \int_{0}^{t}\|\nabla u(\tau)\|_{\infty} d \tau .
$$

Submitting (74) into (68) gives

$$
\begin{gathered}
\|n(t)\|_{H^{s}}^{2}+\|c(t)\|_{H^{s+1}}^{2}+\|u(t)\|_{H^{s+1}}^{2}+\int_{0}^{t}\left(\|n(\tau)\|_{H^{s+1}}^{2}+\|c(\tau)\|_{H^{s+2}}^{2}\right) d \tau \\
\leq \operatorname{Cexpexp}\left(\int_{0}^{t} 1+\|\nabla u(\tau)\|_{\infty}+\|n(\tau)\|_{\infty}^{2}\right) d \tau
\end{gathered}
$$

On the other hand, using the inhomogeneous dynamic partition of the unity, we have

$$
\begin{aligned}
\|\nabla u\|_{L^{\infty}} & =\left\|\sum_{j \geq-1} \nabla \Delta_{j} u\right\|_{L^{\infty}} \leq\left\|\nabla \Delta_{-1} u\right\|_{L^{\infty}}+\sum_{j=0}^{\infty}\left\|\nabla \Delta_{j}\right\|_{L^{\infty}} \\
& \leq C\left(\left\|u_{0}\right\|_{L^{2}}+\sum_{j=0}^{\infty}\left\|\Delta_{j} \omega\right\|_{L^{\infty}}\right) \leq C\left(\left\|u_{0}\right\|_{L^{2}}+\|\omega\|_{B_{\infty, 1}^{0}}\right) .
\end{aligned}
$$

Taking the curl to the third equation of (3) implies

$$
\omega_{t}+u \cdot \nabla \omega=\frac{u^{r}}{r} \omega-\operatorname{curl}(n \nabla \phi) .
$$

Using Lemma 6, we obtain

$$
\begin{aligned}
\|\omega\|_{B_{\infty, 1}^{0}} \leq & C\left(\left\|\omega_{0}\right\|_{B_{\infty, 1}^{0}}+\int_{0}^{t}\|\operatorname{curl}(n \nabla \phi)(\tau)\|_{B_{\infty, 1}^{0}}+\left\|\frac{u^{r}}{r} \omega\right\|_{B_{\infty, 1}^{0}}\right) \\
& \times\left(1+\int_{0}^{t}\|\nabla u(\tau)\|_{L^{\infty}} d \tau\right) \\
\leq & C\left(\left\|\omega_{0}\right\|_{B_{\infty, 1}^{0}}+\int_{0}^{t}\|\operatorname{curl}(n \nabla \phi)(\tau)\|_{B_{\infty, 1}^{0}}+\left\|\frac{u^{r}}{r}\right\|_{L^{\infty}}\|\omega\|_{B_{\infty, 1}^{0}}\right) \\
& \times\left(1+\int_{0}^{t}\|\nabla u(\tau)\|_{L^{\infty}} d \tau\right) .
\end{aligned}
$$

For the term $\|\operatorname{curl}(n \nabla \phi)(\tau)\|_{B_{\infty, 1}^{0}}$, using Bony's decomposition, we have

$$
\begin{aligned}
& \|\operatorname{curl}(n \nabla \phi)(\tau)\|_{B_{\infty, 1}^{0}}=\sum_{\left|q-q^{\prime}\right| \leq 4} 2^{q}\left\|\Delta_{q}\left(S_{q}{ }^{\prime-1} n \Delta_{q}^{\prime} \nabla \phi\right)\right\|_{L^{\infty}} \\
& +\sum_{\left|q-q^{\prime}\right| \leq 4} 2^{q}\left\|\Delta_{q}\left(S_{q}{ }^{\prime-1} \nabla \phi \Delta_{q}{ }^{\prime} n\right)\right\|_{L^{\infty}} \\
& +\sum_{\left|q^{\prime \prime}-q^{\prime}\right| \leq 1} 2^{q}\left\|\Delta_{q}\left(\Delta_{q}{ }^{\prime} n \Delta_{q}{ }^{\prime \prime} \nabla \phi\right)\right\|_{L^{\infty}} \triangleq I_{1}+I_{2}+I_{3} . \\
& q^{\prime} \geq q-3
\end{aligned}
$$

For the term $I_{1}$, we have

$$
I_{1} \leq C \sum_{\left|q-q^{\prime}\right| \leq 4} \sum_{j \leq q^{\prime}-2} 2^{q}\left\|\Delta_{q}^{\prime} \nabla \phi\right\|_{L^{\infty}}\left\|\Delta_{j} n\right\|_{L^{\infty}} \leq C\|\nabla \phi\|_{B_{\infty, \infty}^{1}}\|n\|_{B_{\infty, 1}^{0}} .
$$

Similarly,

$$
I_{2} \leq C\|\nabla \phi\|_{B_{\infty, \infty}^{1}}\|n\|_{B_{\infty, 1}^{0}} .
$$

As for $I_{3}$,

$$
\begin{aligned}
I_{3} \leq C \sum_{\substack{\left|q^{\prime \prime}-q^{\prime}\right| \leq 1 \\
q^{\prime \prime} \geq q-3}} 2^{q-q^{\prime \prime}} 2^{q^{\prime \prime}}\left\|\Delta_{q}^{\prime \prime} \nabla \phi\right\|_{L^{\infty}}\left\|\Delta_{q}^{\prime} n\right\|_{L^{\infty}} \\
\leq C\|\nabla \phi\|_{B_{\infty, \infty}^{1}}\|n\|_{B_{\infty, 1}^{0}} .
\end{aligned}
$$

Plugging (80)-(82) into (79) yields

$$
\|\operatorname{curl}(n \nabla \phi)(\tau)\|_{B_{\infty, 1}^{0}} \leq C\|\nabla \phi\|_{B_{\infty, \infty}^{1}}\|n\|_{B_{\infty, 1}^{0}} .
$$

Putting (83) into (78), using the fact $\left\|u^{r}(t) / r\right\|_{L^{\infty}} \leq C \| \omega_{0}$ $\mid r \|_{L^{3,1}}$ [25] and Gronwall's inequality, we have

$$
\|\omega\|_{B_{\infty, 1}^{0}} \leq C\left(\left\|\omega_{0}\right\|_{B_{\infty, 1}^{0}}+\int_{0}^{t}\|n(\tau)\|_{B_{\infty, 1}^{0}} d \tau\right) e^{\int_{0}^{t}\|\nabla u(\tau)\|_{L^{\infty}} d \tau} .
$$

Substituting (84) into (76) gives

$$
\|\nabla u\|_{L^{\infty}} \leq C\left(\left\|u_{0}\right\|_{H^{s}}+\int_{0}^{t}\|n(\tau)\|_{B_{\infty, 1}^{0}} d \tau\right) e^{\int_{0}^{t}\|\nabla u(\tau)\|_{L^{\infty}} d \tau} .
$$

Applying the Gronwall inequality, we get

$$
\left.\|\nabla u\|_{L^{\infty}} \leq C e^{\exp \left(C \int_{0}^{t}\|n(\tau)\|_{B_{\infty}^{0}, 1}\right.}\right) .
$$

Substituting (86) into (75) and using the fact $B_{\mathrm{o}, 1}^{0}{ }^{\circ} L^{\infty}$, we obtain the desired result.

This completes the proof of Theorem 1.

\section{Data Availability}

Data sharing is not applicable to this article as no data sets were generated or analyzed during the current study.

\section{Conflicts of Interest}

The authors declare that they have no competing interests.

\section{Authors' Contributions}

All authors completed the paper together. All authors read and approved the final manuscript. 


\section{Acknowledgments}

Q. Zhang was partially supported by the National Natural Science Foundation of China (grant numbers 11501160 and 11771423), Natural Science Foundation of Hebei Province (grant numbers A2017201144 and A2020201014), Young Talents Foundation of Hebei Education Department (grant number BJ2017058), and the Second Batch of Young Talents of Hebei Province. This study was supported by the Young Foundation of the Education Department of Hebei Province (grant number QN2020124)" behind "grant number BJ2017058".

\section{References}

[1] A. Kiselev and L. Ryzhik, "Biomixing by chemotaxis and enhancement of biological reactions," Communications in Partial Differential Equations, vol. 37, no. 2, pp. 298-318, 2012.

[2] A. Kiselev and L. Ryzhik, "Biomixing by chemotaxis and efficiency of biological reactions: the critical reaction case," Journal of Mathematical Physics, vol. 53, no. 11, pp. 298-318, 2012.

[3] J. C. Coll, B. F. Bowden, G. V. Meehan et al., "Chemical aspects of mass spawning in corals. I. Sperm-attractant molecules in the eggs of the scleractinian coral Montipora digitata," Marine Biology, vol. 118, no. 2, pp. 177-182, 1994.

[4] J. C. Coll, P. A. Leone, B. F. Bowden et al., "Chemical aspects of mass spawning in corals. II. (-)-Epi-thunbergol, the sperm attractant in the eggs of the soft coral Lobophytum crassum (Cnidaria:Octocorallia)," Marine Biology, vol. 123, no. 1, pp. 137-143, 1995.

[5] R. L. Miller, "Sperm chemotaxis in the hydromedusae. I. Species-specificity and sperm behavior," Marine Biology, vol. 53, no. 2, pp. 99-113, 1979.

[6] R. L. Miller, "Demonstration of sperm chemotaxis in Echinodermata: Asteroidea, Holothuroidea, Ophiuroidea," Journal of Experimental Zoology, vol. 234, no. 3, pp. 383-414, 1985.

[7] M. Di Francesco, A. Lorz, and P. Markowich, "Chemotaxis fluid coupled model for swimming bacteria with nonlinear diffusion: global existence and asymptotic behavior," Discrete \& Continuous Dynamical Systems - A, vol. 28, no. 4, pp. 1437$1453,2010$.

[8] J. G. Liu and A. Lorz, "A coupled chemotaxis-fluid model: global existence," Annales de l'Institut Henri Poincaré C, Analyse Non Linéaire, vol. 28, no. 5, pp. 643-652, 2011.

[9] A. Lorz, "A coupled Keller-Segel-Stokes model: global existence for small initial data and blow-up delay," Communications in Mathematical Sciences, vol. 10, no. 2, pp. 555-574, 2012.

[10] Y. Tao and M. Winkler, "Global existence and boundedness in a Keller-Segel-Stokes model with arbitrary porous medium diffusion," Discrete \& Continuous Dynamical Systems - A, vol. 32, no. 5, pp. 1901-1914, 2012.

[11] M. Winkler, "Global large-data solutions in a chemotaxis(Navier-)Stokes system modeling cellular swimming in fluid drops," Communications in Partial Differential Equations, vol. 37, no. 2, pp. 319-351, 2012.

[12] Q. Zhang and X. Zheng, "Global well-posedness for the twodimensional incompressible chemotaxis-Navier-Stokes equations," SIAM Journal on Mathematical Analysis, vol. 46, no. 4, pp. 3078-3105, 2014.
[13] Q. Zhang, "Local well-posedness for the chemotaxis-NavierStokes equations in Besov spaces," Nonlinear Analysis: Real World Applications, vol. 17, pp. 89-100, 2014.

[14] Q. Zhang and P. Wang, "Global well-posedness for the 2D incompressible four-component chemotaxis- Navier-Stokes equations," Journal of Differential Equations, vol. 269, no. 2, pp. 1656-1692, 2020.

[15] Q. Zhang and X. Zheng, "Global well-posedness of axisymmetric solution to the $3 \mathrm{D}$ axisymmetric chemotaxis-Navier-Stokes equations with logistic source," Journal of Differential Equations, vol. 274, pp. 576-612, 2021.

[16] J. Lankeit, "Long-term behaviour in a chemotaxis-fluid system with logistic source," Mathematical Models and Methods in Applied Sciences, vol. 26, no. 11, pp. 2071-2109, 2016.

[17] J. Beale, T. Kato, and A. Majda, "Remarks on the breakdown of smooth solutions for the 3-D Euler equations," Communications in Mathematical Physics, vol. 94, no. 1, pp. 61-66, 1984.

[18] Q. Chen, C. Miao, and Z. Zhang, “A new Bernstein's inequality and the 2D dissipative quasi-geostrophic equation," Communications in Mathematical Physics, vol. 271, no. 3, pp. 821838, 2007.

[19] Q. Chen, C. Miao, and Z. Zhang, "On the well-posedness of the ideal MHD equations in the Triebel-Lizorkin spaces," Archive for Rational Mechanics and Analysis, vol. 195, no. 2, pp. 561$578,2010$.

[20] Q. Zhang, "Blowup criterion of smooth solutions for the incompressible chemotaxis-Euler equations," Zeitschrift für Angewandte Mathematik und Mechanik, vol. 96, no. 4, pp. 466-476, 2016.

[21] Q. Zhang, "On the inviscid limit of the three dimensional incompressible chemotaxis- Navier-Stokes equations," Nonlinear Analysis: Real World Applications, vol. 27, pp. 70-79, 2016.

[22] C. Miao, J. Wu, and Z. Zhang, Littlewood-Paley Theory and Applications to Fluid Dynamics Equations. Monographs on Modern Pure Mathematics, No. 142, Science Press, Beijing, 2012.

[23] R. Danchin, "A few remarks on the Camassa-Holm equation," Differential and Integral Equations, vol. 14, no. 8, pp. 953-988, 2001.

[24] T. Hmidi and S. Keraani, "Incompressible viscous flows in borderline Besov spaces," Archive for Rational Mechanics and Analysis, vol. 189, no. 2, pp. 283-300, 2008.

[25] H. Abidi, T. Hmidi, and S. Keraani, "On the global wellposedness for the axisymmetric Euler equations," Mathematische Annalen, vol. 347, no. 1, pp. 15-41, 2010. 